\title{
Sexuelle Orientierung im therapeutischen Kontext. Thematische Besonderheiten in der Beziehungsgestaltung mit homo- und bisexuellen Patient*innen
}

\author{
Claudia Höfner · Kai Ginkel · Gerald Käfer-Schmid
}

Online publiziert: 10. Mai 2019

(c) Der/die Autor(en) 2019

\begin{abstract}
Zusammenfassung Die Sozialisation homo- und bisexueller Menschen birgt innerhalb der heteronormativen Mehrheitsgesellschaft einige Besonderheiten, die in Bezug auf die therapeutische Beziehung reflektiert werden müssen. Sie sehen sich häufig Erfahrungen von Stress, Problematisierung und Diskriminierung ausgesetzt. Es fehlt überdies oft an positiven Rollenvorbildern. Der vorliegende Beitrag reflektiert diese Besonderheiten etwa in Bezug auf das Comingout als lebenslangen Prozess oder die Überwindung einer oftmals internalisierten Homonegativität. Hierbei steht als Zielsetzung der Einsatz für eine bessere, barriere- und diskriminierungsarme Gesundheitsversorgung und Behandlung durch kompetente und wertschätzende Fachkräfte im Mittelpunkt.
\end{abstract}

Schlüsselwörter Therapeutische Beziehung •

Homosexualität · Therapieempfehlungen ·

Diskriminierung · Gender Studies

Sexual orientation in psychotherapy. On the specifics of the therapeutic relation with homosexual and bisexual patients

Summary Within heteronormative society, the socialization of homosexual and bisexual people harbors some peculiarities that should be reflected with regard to the therapeutic relation. Homosexual and bisexual people are often exposed to experiences of stress, problematisation, and discrimination. There is also a lack of positive role models. This article reflects on these specifics, e.g. with regard to "coming out" as a lifelong process and overcoming an often internalized homonegativity. The focus here is on the devel-

C. Höfner $(\bowtie)$

Sensengasse 7/8, 1090 Wien, Österreich

praxis@claudiahoefner.at opment of better, accessible and non-discriminatory health care and treatment by competent and appreciative professionals.

Keywords Therapeutic relation · Homosexuality • Guidelines and recommenations - Discrimination · Gender studies

\section{Einleitung: Gleich und doch anders}

Lesbische, schwule und bisexuelle Menschen sind aufgrund ihres Minderheitenstatus in einer heteronormativen Mehrheitsgesellschaft sozialisiert und machen andere Erfahrungen von Diskriminierung und Privilegierung als Heterosexuelle. Sie durchleben eine sexuelle Identitätsentwicklung, bei der sie ihre internalisierte Homonegativität überwinden müssen. Sie sind als Minderheit überdies spezifischen Risikodynamiken und Stressfaktoren ausgesetzt (Göth und Kohn 2014, S. V). Diese variieren je nach Kultur und Epoche, denn homo- und bisexuelle Personen machen unterschiedliche Erfahrungen mit Definitionen und Auffassungen von Sexualverhalten, Geschlechterrollen und sexuellen Orientierungen. Aus diesem Grund macht es Sinn, den Blick auf die therapeutische Beziehung in der Behandlung dieser Menschen zu schärfen - auch wenn dieser Versuch immer wieder die Gefahr einer erneuten Diskriminierung birgt (Rauchfleisch et al. 2002).

Das populäre Konzept der sexuellen Orientierung birgt viele stereotype Vorstellungen in sich, die eng an das biologische („sex“) wie auch an das soziale Geschlecht (,gender“) geknüpft sind, und bleibt innerhalb des binären Geschlechtscodes verhaftet (Butler 1991, S. 22). Daher kann es im Falle von Abweichungen zu einer „Therapeutisierung“ oder einer gesellschaftlichen Disziplinierung kommen (Maasen 2010). Bei abweichendem Begehren betreffen diese Diszipli- 
nierungen etwa Sanktionierungen der nicht befolgten Praktiken der Zweigeschlechtlichkeit, es werden Ängste und Schuldgefühle geschürt, Gefahren behauptet.

Gleichgeschlechtliche Orientierungen haben selbst nichts mit psychischer Gesundheit oder Krankheit zu tun, sondern enthalten - ebenso wie die heterosexuellen Ausrichtungen - das gesamte Spektrum von Gesundheit und Krankheit (Rauchfleisch 2002a). „Da die Bedingungen, unter denen Lesben, Schwule und Bisexuelle trotz zunehmender Akzeptanz in unserer Gesellschaft leben, nach wie vor jedoch mehr oder weniger große Schwierigkeiten bieten (...), kann es bei ihnen zu Anpassungsstörungen kommen, die nicht auf eine primäre psychische Störung zurückzuführen sind, sondern Folgen ihrer spezifischen - belastenden Lebenssituation sind“ (Rauchfleisch 2002a, S. 37). Dabei müsse man sich gewahr sein, dass sich gleichgeschlechtlich empfindende Menschen mit psychischen Störungen im Vergleich zu heterosexuellen psychisch Erkrankten in einer schwierigeren Lage befinden, da bei Heterosexuellen die sexuelle Orientierung nie in einen ursächlichen Zusammenhang mit ihren psychischen Problemen gebracht wird.

In diesem Artikel geht es daher um Themen wie bisher erlittene Verletzungen, fehlende Rollenmodelle, Coming-out, verinnerlichte Homophobie und Homonegativität, spezielle Belastungen im Alltag und Besonderheiten in der Paardynamik und -struktur, wie die Verdoppelung der Geschlechtsrollen oder der Umgang mit Unterschieden.

\section{Fehlende Rollenmodelle}

Die heteronormative Mehrheitsgesellschaft bietet uns eine Vielfalt von heterosexuellen Vorbildern und Modellen an, die Anleitungen dafür geben, wie man sich als Mädchen oder Bub, als Mann oder Frau verhält, wenn man verliebt ist, wie man flirtet, wie man Sexualität lebt oder Beziehungen gestaltet. Auch wenn viele dieser Bilder, Normen und Werte, die junge Menschen über Schönheitsideale, Körperbilder und Sexualität medial vermittelt bekommen, kritisch betrachtet werden müssen, bleiben sie in ihrem Kern in Bezug auf Heterosexualität stimmig. Sie werden lange vor der Adoleszenz internalisiert und erzeugen in der Regel eine von Umwelt und Individuum geteilte Erwartung, sich heterosexuell zu entwickeln. Verläuft die Entwicklung anders als erwartet, machen homosexuelle Menschen die Erfahrung, dass die heteronorme Gesellschaft Homo- und Bisexualität nicht als gleichwertige, normale Entwicklung berücksichtigt, sondern oftmals abwertet oder problematisiert (Göth und Kohn 2014, S. 23).

Die fehlenden Identifikationsfiguren und Leitbilder (Roth 2002, S. 130) sowie die Unsichtbarkeit gleichgeschlechtlicher Beziehungs- und Familienmodelle verstärkt die Unsicherheit vieler Menschen mit gleichgeschlechtlichen Beziehungswünschen, welche unrealistisch und verboten erscheinen (Göth und
Kohn 2014, S. 162). Homo- und bisexuelle Menschen sehnen sich daher im Stadium der Entdeckung ihrer individuellen Identität nach „idealen“ (ebenfalls homo- oder bisexuellen) Psychotherapeut*innen, die sie an der sicheren Hand nehmen. Diesen Therapeut*innen können selbst noch nicht erreichte Entwicklungsziele - und im Sinne einer idealisierenden Übertragung ein erfolgreiches Coming-out sowie Erfolg im beruflichen wie privaten Alltag in allen möglichen Facetten - unterstellt werden: erfolgreiche Bewältigungsstrategien gegenüber Diskriminierung, ein glückliches Beziehungsgefüge inklusive gelebter Intimität und Sexualität (ebd.).

Im therapeutischen Kontext stellt sich folglich die Frage nach der Deklaration der sexuellen Orientierung der Therapeut*in gegenüber der Patient*in. Eine selektive Offenheit der Psychotherapeut*in in Bezug auf die eigene sexuelle Orientierung ist daher eine Option, auch wenn dies wohl überlegt sein muss (Wiesendanger 2002, S. 110). Es kommt mitunter vor, dass homosexuelle Patient*innen nach der sexuellen Orientierung der Psychotherapeut*in fragen. Frossard (2002b, S. 173) findet es „durchaus sinnvoll, eine solche Frage, nach einer fundierten Abklärung ihrer tieferen Ursache, zu beantworten“. Viele Patient*innen haben sich ohnehin gut über ihr Gegenüber informiert. Rauchfleisch (2001) weist darauf hin, dass die Frage nach der sexuellen Präferenz oft eine Auseinandersetzung mit dem Trennenden - oder gegebenenfalls dem Verbindenden - in der therapeutischen Beziehung mit sich bringt. Insofern könnte das Schweigen der Psychotherapeut*in als Verschweigen oder Scham interpretiert werden, was einem negativen Vorbild gleichkäme.

Auf der einen Seite erleichtert eine Offenlegung der gleichgeschlechtlichen Orientierung der Therapeut*in es der Patient*in, ihrem Gegenüber breites Wissen und Selbsterfahrung hinsichtlich homosexueller Identität sowie bedingungslose Akzeptanz und Wertschätzung zu unterstellen und sich somit mit ihrer ganzen Persönlichkeit zu zeigen, ohne Gefahr zu laufen, sich rechtfertigen $\mathrm{zu}$ müssen (Wiesendanger 2002, S. 110). Patient*innen finden sich - im Unterschied zu einer überwiegend ablehnend gestimmten Alltagswelt - in einer zunächst wohlwollenden und konfliktfreien therapeutischen Atmosphäre wieder, zumindest was ihre sexuelle Identität und Orientierung anbelangt (Roth 2002, S. 135). Homosexuelle Psychotherapeut*innen können in diesem Fall durch verschiedenste Teilidealisierungen zum notwendigen Identifikationsobjekt werden. Patient*innen „können im weiteren Behandlungsverlauf die zunächst an ihrem Gegenüber realen oder phantasierten Eigenschaften und Wertvorstellungen der lesbischen, schwulen und bisexuellen Individuation schrittweise verinnerlichen und weiterentwickeln“ (ebd., S. 130).

Auf der anderen Seite könnte die Therapeut*in bei Offenlegung ihrer Homosexualität zur Projektionsfläche für widerstreitende, auch homophobe Gefühle der Patient*in werden (Wiesendanger 2002, S. 110). Wei- 
ters besteht das Risiko, dass durch eine überempathische Haltung die notwendige Distanz verlorengeht, wodurch sich Psychotherapeut*in wie Patient*in im gleichen Boot sitzend wiederfinden und die tendenziell als feindselig erlebte heterosexuelle Welt ausschließen und im Außen festmachen (Roth 2002, S. 135).

\section{Coming-out, ein lebenslanger Prozess}

Im heteronormativen Kontext ist eine Besonderheit in der Biographie homo- und bisexueller Menschen der Entwicklungsprozess des Coming-out, bestehend aus verschiedenen Phasen. Das Coming-out umfasst einerseits einen innerpsychischen Vorgang, also das Gewahrwerden sowie die anschließende Gewissheit, nicht heterosexuell zu sein (Rauchfleisch 2002b, S. 38), andererseits eine soziale Dimension, nämlich die schrittweise Veröffentlichung der eigenen nichtheterosexuellen Identität in einer heteronormativen Gesellschaft (Göth und Kohn 2014, S. 23) sowie das Finden eines eigenen Lebensstils. Es wird hierbei zwischen der inneren Auseinandersetzung und dem Benennen und Aussprechen der Gefühle und Erkenntnisse unterschieden, was sich gegenseitig beeinflusst (ebd.).

Bewusst gemacht werden sollte in jeder Psychotherapie das Prozesshafte am Coming-out: Es handelt sich letztlich um einen lebenslangen Prozess, der durch seine sozialen Aspekte besondere Belastungen mit sich bringen kann (Rauchfleisch 2002b, S. 38). Fortlaufend fallen Entscheidungen an, wie man sich in welchem Kontext präsentiert: „Sich verändernde Bedingungen und Lebensereignisse, z. B. Gesetzesänderungen, neue Beziehungskonstellationen, Migration, Arbeitsplatzwechsel, Ortswechsel (Stadt-Land, Ost-West u.Ä.), erlebte und sich ändernde Diskriminierungen und Privilegierung setzen immer wieder innerpsychische Prozesse in Gang und machen erneute Anpassungsleistungen notwendig“ (Göth und Kohn 2014, S. 22).

\section{Internalisierte Homonegativität}

Ein weiteres - bisweilen übersehenes - Konfliktfeld ist internalisierte Homonegativität. Darunter wird die Verinnerlichung „von soziokulturell vorgegebenen negativen Einstellungen und Bildern durch nicht-heterosexuell empfindende Menschen“ verstanden (Göth und Kohn 2014, S. 19). Das Modell setzt sowohl bei gesellschaftlichen Bedingungen als auch beim individuellen Erleben an und beginnt bereits in der Kindheit zu wirken (Rauchfleisch et al. 2002; Fiedler 2004). Hegemoniale Werte und Normen vermitteln sich dabei nicht nur über direkte Benennungen, sondern auch über Auslassungen und Verschweigen. Diese vielfältigen negativen Bilder und Einstellungen können von vielen nicht-heterosexuellen Menschen übernommen und häufig beim ersten Verlieben oder der Nähe von homosexuellen Menschen aktualisiert werden (Göth und Kohn 2014, S. 19). Laut Wiesendanger (2005, S. 5) handelt es sich dabei auch um ,alltägliche Traumatisierungen durch verinnerlichte Homophobie“. Göth und Kohn (2014, S. 19) sprechen überdies von verletzenden und invalidisierenden Erfahrungen durch die Selbstverständlichkeit, mit der über homosexuelle Menschen gelacht, gespottet, gewitzelt wird bzw. ihr Vorhandensein geleugnet, tabuisiert oder schamhaft abgewehrt wird.

Nicht immer können sich Patient*innen an konkrete Diskriminierungs- und Gewalterfahrungen in ihrer Kindheit erinnern, viel häufiger beschreiben sie Gefühle von Unbehagen, Vermeiden-Wollen oder Anders-Sein (Göth und Kohn 2014, S. 20). Rauchfleisch (2002b, S. 40) meint, dass schon „die - verständliche - Erwartung heterosexueller Eltern, ein heterosexuelles Kind vor sich zu haben, (...) im Heranwachsenden schmerzliche Gefühle des Nicht-verstanden-Werdens und des Ausgeschlossen-Seins entstehen lassen“ kann. Je tiefer diese frühkindlichen innerpsychischen Verletzungen sind, desto nachhaltiger wird das Kind in einem zentralen identitätsstiftenden Bereich destabilisiert und erlebt Entfremdung und Verwirrung (Wiesendanger 2002, S. 68). In einem sekundären Prozess „verhärtet sich das Ganze, wobei von außen induzierte, stark selbstwertmindernde Scham- und Schuldgefühle hinzukommen" (ebd.). Das Kind ist gezwungen, die eigene Homo- bzw. Bisexualität abzuwehren, $\mathrm{zu}$ verdrängen, abzuspalten, was im Jugend- und Erwachsenenalter zu einer Fülle unterschiedlicher psychischer und psychosomatischer Störungsbilder führen kann. Auch zu einer Identifikation mit dem Aggressor kann es kommen (ebd., S. 69).

Bisweilen regt bereits die Identifikation internalisierter Homonegativität Veränderungsprozesse an, da in der Reflexion deutlich wird, dass die aufgespürten homonegativen Bewertungen in Konflikt $\mathrm{zu}$ anderen Überzeugungen stehen. Doch manchmal bleiben die Bewertungen trotz kognitiver Dissonanz bestehen (Göth und Kohn 2014, S. 146). Um solche selbstblockierenden Verhaltensweisen und Denkstrukturen auflösen zu können, müssen sich Therapeut*innen über das Ausmaß sowie die Tragweite innerer und äußerer Homophobie bewusst sein und diese auch gezielt identifizieren können (Frossard 2002b, S. 171). Internalisierte Homonegativität kann sich auch subtil ausdrücken. Mögliche Anzeichen hierfür sind: Wenn Patient*innen das Wort „schwul“ oder „lesbisch“ vermeiden, Kontakte $\mathrm{zu}$ anderen gleichgeschlechtlich liebenden Menschen vermeiden, sich negativ über die Community äußern (ebd.). Es lohnt sich, nachzufragen, wie viele Personen in der Arbeit oder im Freundeskreis Bescheid wissen, wie und wann sie ihre Familie informiert haben und wie sie dies erlebt haben (ebd.). 
Verdoppelung der Geschlechtsrollen und der Umgang mit Unterschieden

Dynamiken in homosexuellen Paarbeziehungen unterscheiden sich kaum von jenen in heterosexuellen. In beiden Formen gibt es ein breites Spektrum an Spielarten und Auseinandersetzungen. Dennoch ist bei Paartherapien folgendes zu bedenken: Erstens fehlt bei gleichgeschlechtlich liebenden Menschen oft die gesellschaftliche Anerkennung, zweitens besteht das Paar aus zwei Personen, die gleich sozialisiert wurden (Frossard 2002a, S. 141). Laut Göth und Kohn (2014, S. 165) beschreiben viele gleichgeschlechtliche Paare eine große Verbundenheit und Nähe, was „nach Zeiten und Erfahrungen der Isolation und Einsamkeit während der Entwicklung der sexuellen Identität eine besonders schöne und stärkende Erfahrung" darstellt. Durch stark prägende Geschlechterrollen ähneln sich viele Sozialisationserlebnisse „und auch manche Art des Denkens und Fühlens“ (ebd.). Dies kommt einer Verdoppelung der Geschlechtsrollen gleich, die sich auch auf den Umgang mit vorhandenen Unterschieden auswirkt.

Man darf aus therapeutischer Sicht nicht in klischeehafte Interpretationen von symbiotischen Frauenbeziehungen und promiskuitiven Männerbeziehungen verfallen. Zwar kommt es durchaus vor, dass lesbische Paare in einer Beziehung mit symbiotischen Zügen leben oder dass schwule Beziehungen offen gestaltet sind und Sexualität auch außerhalb der Partnerschaft stattfindet. „Ob und inwieweit diese Phänomene bei lesbischen bzw. schwulen Paaren tatsächlich häufiger (oder allenfalls auch nur nach außen deutlicher sichtbar bzw. dem Partner/der Partnerin gegenüber offener kommuniziert) auftreten als bei heterosexuellen Paaren", bedarf weiterführender Forschung (Frossard 2002a, S. 141). Immerhin belegen einige Studien (z.B. Huse und Watzlawik 2009; Seferović 2003) eine hohe Zufriedenheit homosexueller Paare, teilweise sogar höher als bei heterosexuellen.

Seferović (2006) weist in Bezug auf schwule Partnerschaften darauf hin, dass es im Unterschied zu heterosexuellen Paaren einen Zusammenhang zwischen Partnerschaftszufriedenheit und der Gleichverteilung von Macht gibt. Was das Konzept der sexuellen Nicht-Exklusivität anbelangt, so zeigen die Ergebnisse seiner Studie, dass viele schwule Paare diese akzeptieren. Wesentlich für die partnerschaftliche Zufriedenheit scheinen transparente Abmachungen zu sein, bestehend aus konkreten und praktikablen Vereinbarungen hinsichtlich des Sexualverhaltens. Psychotherapeut*innen müssen daher wissen, „dass es keinerlei normative Partnerschaftsmodelle gibt, die in der so genannten Szene favorisiert würden. Bereits das, was schwule Männer unter ,Treue` verstehen oder unter , außerpartnerschaftlichem Sex' unterscheidet sich von Paar zu Paar. Hier ist es für die Therapie essenziell, sich diese Konstrukte operational beschreiben zu lassen“ (Hammelstein 2007).
Berlage (2001) hat sich mit symbiotischen lesbischen Beziehungen beschäftigt und meint, dass die Fähigkeit zu Nähe und Empathie Teil der weiblichen Sozialisation ist. Diese Eigenschaften können dazu verführen, „in der Beziehung“ und den realen bis ,angenommenen Wünschen der anderen aufzugehen“, teils indem eigene Wünsche zurückgestellt werden, bis das eigene „Selbstwertgefühl dadurch brüchig geworden ist" (Göth und Kohn 2014, S. 165). Frossard (2002a) meint, dass vielfach auftretende Konflikte durch das Spannungsfeld von Nähe und Distanz entstehen und durch die weibliche Sozialisation zwar einerseits ein eher beziehungserhaltendes Konfliktverhalten aufweisen, andererseits die eigenen Bedürfnisse zu wenig wahrnehmen oder artikulieren. Auch kann es sein, dass aufgrund des hohen Wertes der Gleichberechtigung Machtunterschiede innerhalb der Beziehung tabuisiert werden (ebd.).

Neben Problemen, die auch heterosexuelle Paare in ihren Beziehungen erleben, gibt es in homosexuellen Partnerschaften zusätzliche Besonderheiten und Herausforderungen, die jedoch auch andere Perspektiven, neue Wege und Freiheiten erlauben (Göth und Kohn 2014, S. 162). Da gleichgeschlechtlich liebende Menschen nahezu ausschließlich mit heterosexuellen Partnerschaftsmodellen heranwachsen, fehlen oft passende Modelle, wenn sie ihre ersten homosexuellen Beziehungen eingehen. Dies bietet Risiko und Chance zugleich. Ein Vorteil dieser Modelllosigkeit liegt etwa im Fehlen tradierter Rollenmuster geschlechtsspezifischer Arbeitsteilung (Re/Produktion). Diese muss erst individuell ausgehandelt werden (Hammelstein 2007). Andererseits kann das hohe Maß an geteilten Erfahrungen „Paare und Beziehungsnetzwerke dazu bringen, ihre individuellen Unterschiede nicht ausreichend wahrzunehmen und/oder nicht wertschätzen zu können“ (Göth und Kohn 2014, S. 167).

Die Folien traditionell heterosexueller Partnerschaftsmodelle lassen sich also nicht eins zu eins über homo- oder bisexuelle Beziehungen legen. Aufgrund der immer noch besonderen Lebensumstände „leben Lesben und Schwule ihre Partnerschaften in der Regel egalitärer und benötigen in dieser auch ein deutlicheres Ausmaß an Autonomie" (Wahala 2007, S. 13). Psychotherapeut*innen fehlt es hier nicht selten an Kenntnissen über gleichgeschlechtliche Partnerschaftsmodelle (ebd.).

\section{Fazit}

Homo- und bisexuelle Menschen können das gesamte Spektrum psychischer Störungen entwickeln. Dies hat aber - mit Ausnahme von Problemen mit internalisierter Homophobie - nichts mit ihrer sexuellen Orientierung zu tun. Stattdessen müssen verschiedene Formen antihomosexueller Gewalt unserer Gesellschaft als gestörte Verhaltensweisen bezeichnet werden. 
Die psychotherapeutische Behandlung homo- und bisexueller Patient*innen war lange geprägt von pathologisierenden und abwertenden Konzeptionen gleichgeschlechtlicher Lebensweisen. Nach der Entpathologisierung in ICD und DSM entwickelte sich laut Wolf et al. (2015, S. 21) „eine Diskrepanz zwischen einer zunehmenden Fülle von affirmativen und die Diversität dieser Bevölkerungsgruppe herausarbeitenden Forschungsarbeiten einerseits und der unzureichenden Integration des Fachwissens in die psychotherapeutische und beratende Praxis andererseits“. Heute wird die Bedeutung eines Verständnisses für den gesellschaftlichen Kontext, in dem gleichgeschlechtlich liebende Menschen leben, für eine fachgerechte Gestaltung von Psychotherapie zunehmend anerkannt (ebd.). ${ }^{1}$

Hinsichtlich der verschiedenen psychotherapeutischen Verfahren ist festzuhalten, dass „jede ,Schule“ spezifische Chancen, teilweise aber auch Gefahren beinhaltet“ und bisweilen „eine von Diskriminierungen und Gewalt geprägte Historie gegenüber homound bisexuellen sowie transidenten Patient_innen“ hat (Wolf und Meyer 2017, S. 136). Es reicht aber nicht aus, sich mit der entsprechenden Fachliteratur zum Thema LSBT*Q (Lesbisch, Schwul, Bisexuell, Transgender, Queer) auseinanderzusetzen, sondern bedarf zusätzlich eines Community-basierten Wissens zur Vorbeugung einer „einseitigen und unvollständigen Wissensbildung“. Dies beinhaltet eine vertiefte Beschäftigung „mit gesellschaftlichen Stigmatisierungsprozessen und eigenen Erfahrungen von Privilegierung und Diskriminierung sowie das bewusste Umlernen von erlernten Vorurteilen“ (Wolf und Meyer 2017, S. 137).

Wir stimmen daher mit Wolf und Meyer (2017, S. 137) überein, dass es wichtig ist, aus der eigenen Professionalität heraus nie zu vergessen, wieviel Leid homo- und bisexuellen Patient*innen im therapeutischen Kontext vielfach zugefügt wurde, und dass Psychotherapeut*innen gegebenenfalls „dazu Stellung $\mathrm{zu}$ beziehen und im therapeutischen Vorgehen sehr transparent zu arbeiten" haben, um ihren $\mathrm{Pa}$ tient*innen Selbstbestimmung zu ermöglichen. Dazu zählt auch, eigene Unsicherheit angemessen zu thematisieren. „Denn gerade auf dem Terrain, auf das uns die Sozialisation innerhalb der heterosexuellen Matrix denkbar schlecht vorbereitet hat, unterlaufen uns Fehler - wobei auch Psychotherapeut_innen, die selbst einen LSBT*Q-Hintergrund haben, nicht frei

\footnotetext{
${ }^{1}$ Um eine „fachlich fundierte, kontextsensible, hilfreiche, Schäden vermeidende Gesundheitsversorgung von Lesben, Schwulen und Bisexuellen zu fördern“, haben Wolf et al. (2015, S. 22) evidenzbasierte „Empfehlungen zur Psychotherapie und Beratung mit lesbischen, schwulen und bisexuellen Klient_innen“ herausgegeben, deren konkrete Inhalte den Rahmen sprengen würden. Davor hat schon die American Psychological Association (APA) im Jahr 2011 „Guidelines for Psychological Practice With Lesbian, Gay, and Bisexual Clients“ veröffentlicht, die Grundlagenwissen vermitteln und auch auf intersektionale Aspekte eingehen.
}

davon sind“ (ebd.). Es ist von Bedeutung, sich aus der soziokulturell machtvollen und privilegierten Position als Therapeut*in heraus sowohl individuell als auch berufspolitisch für die Belange von LSBT*Q-Personen einzusetzen und für eine bessere, barriere- und diskriminierungsarme Gesundheitsversorgung und Behandlung durch kompetente und wertschätzende Fachkräfte zu engagieren.

Interessenkonflikt C. Höfner, K. Ginkel und G. Käfer-Schmid geben an, dass kein Interessenkonflikt besteht.

Open Access Dieser Artikel wird unter der Creative Commons Namensnennung 4.0 International Lizenz (http:// creativecommons.org/licenses/by/4.0/deed.de) veröffentlicht, welche die Nutzung, Vervielfältigung, Bearbeitung, Verbreitung und Wiedergabe in jeglichem Medium und Format erlaubt, sofern Sie den/die ursprünglichen Autor(en) und die Quelle ordnungsgemäß nennen, einen Link zur Creative Commons Lizenz beifügen und angeben, ob Änderungen vorgenommen wurden.

\section{Literatur}

American Psychological Assiciation (APA) (2011). Guidelines for psychological practice with lesbian, gay, and bisexual clients. American Psychologist, 67(1), 10-42. https:// www.apa.org/pubs/journals/features/amp-a0024659. pdf [06.01.2019].

Berlage, K. (2001). Paardynamik in lesbischen Beziehungen. In Deutsche Aids-Hilfe (Hrsg.), Beratung von Lesben und Schwulen. Berlin: DeutscheAids-Hilfe.

Butler, J. (1991). Das Unbehagen der Geschlechter. Gender Studies. Frankfurt am Main: Suhrkamp.

Fiedler, P. (2004). Sexuelle Orientierung und sexuelle Abweichung. Weinheim: Beltz.

Frossard, J. (2002a). Lesbische Paare. In U. Rauchfleisch, J. Frossard, G. Waser, K. Wiesendanger \& W. Roth (Hrsg.), Gleich und doch anders. Psychotherapie und Beratung von Lesben, Schwulen, Bisexuellen und ihren Angehörigen (S. 141-151). Stuttgart: Klett-Cotta.

Frossard, J. (2002b). Irrwege und Auswege in Psychotherapie und Beratung lesbischer, schwuler und bisexueller Klientinnen und Klienten. In U. Rauchfleisch, J. Frossard, G. Waser, K. Wiesendanger \& W. Roth (Hrsg.), Gleich und doch anders. Psychotherapie und Beratung von Lesben, Schwulen, Bisexuellen und ihren Angehörigen (S. 169-176). Stuttgart: Klett-Cotta.

Göth, M., \& Kohn, R. (2014). Sexuelle Orientierung in Psychotherapie und Beratung. Berlin, Heidelberg: Springer.

Hammelstein, P. (2007). Psychotherapeutisches Arbeiten mit schwulen Männern. Ein Beitrag zur Diskussion. Verhaltenstherapie \& Psychosoziale Praxis, 39. https://www.dgvt-fortbildung.de/interaktivefortbildung/archiv-der-fachartikel/archiv/hammelsteinp-2007-psychotherapeutisches-arbeiten-mit-schwulenmaennern-ein-beitrag-zur-diskussion/. Zugegriffen: 28 . Jan. 2019.

Huse, A., \& Watzlawik, M. (2009). Sind Lesben die treueren Seelen? In M. Watzlawik \& N. Heine (Hrsg.), Sexuelle Orientierungen. Weg vom Denken in Schubladen (S. 67-78). Göttingen:Vandenhoeck\& Ruprecht.

Maasen, S. (2010). Zur Therapeutisierung sexueller Selbste. „The Making Of“ einer historischen Diskursanalyse. In R. Keller, A. Hirseland, W. Schneider \& W. Viehöver 
(Hrsg.), Handbuch sozialwissenschaftliche Diskursanalyse(S. 121-148). Wiesbaden:VS.

Rauchfleisch, U. (2001). Schwule, Lesben, Bisexuelle. Lebensweisen, Vorurteile, Einsichten. Göttingen:Vanderhoeck \& Ruprecht.

Rauchfleisch, U. (2002a). Historischer Abriß. In U. Rauchfleisch, J. Frossard, G. Waser, K. Wiesendanger \& W. Roth (Hrsg.), Gleich und doch anders. Psychotherapie und Beratung von Lesben, Schwulen, Bisexuellen und ihren Angehörigen (S. 15-37). Stuttgart: Klett-Cotta.

Rauchfleisch, U. (2002b). Coming-out, ein lebenslanger Prozess. In U. Rauchfleisch, J. Frossard, G. Waser, K. Wiesendanger \&W. Roth (Hrsg.), Gleich und doch anders. Psychotherapie und Beratung von Lesben, Schwulen, Bisexuellen und ihren Angehörigen (S.38-52). Stuttgart: Klett-Cotta.

Rauchfleisch, U., Frossard, J., Waser, G., Wiesendanger, K., \& Roth, W. (Hrsg.). (2002). Gleich und doch anders. Psychotherapie und Beratung von Lesben, Schwulen, Bisexuellen und ihren Angehörigen. Stuttgart: Klett-Cotta.

Roth, W. (2002). Übertragung und Gegenübertragung in der therapeutischen Beziehung. In U. Rauchfleisch, J. Frossard, G. Waser, K. Wiesendanger \& W. Roth (Hrsg.), Gleich und doch anders. Psychotherapie und Beratung von Lesben, Schwulen, Bisexuellen und ihren Angehörigen (S. 124-135). Stuttgart: Klett-Cotta.

Seferović, S. (2003). Völlig gleich oder grundverschieden? Homo- und heterosexuelle Partnerschaften im Vergleich. In M. C. Steffens \& M. Ise (Hrsg.), Jahrbuch Lesben - Schwule - Psychologie. Lengerich: Pabst.
Seferović, S. (2006). Paartherapiemitschwulen Paaren. Verhaltenstherapie \& psychosoziale Praxis, 38,625-641.

Wahala, J. (2007). Homosexualität und Homophobie heute. Eine kritische Konfrontation mit der vorherrschenden Heteronormativität und den daraus resultierenden Vorurteilen und Zerrbildern. Vortrag an der Leopold Franzens Universität Innsbruck, Ringvorlesung zur interdisziplinären Sexualwissenschaft. Verfügbar unter https://www.oegs.or.at/perch/resources/ homosexualitaetundhomophobieheuteinnsbruckpublikation.pdf [10.01.2019].

Wiesendanger, K. (2002). Grundlagen von Psychotherapie und Beratung bei schwulen und bisexuellen Männern. In U. Rauchfleisch, J. Frossard, G. Waser, K. Wiesendanger \& W. Roth (Hrsg.), Gleich und doch anders. Psychotherapie und Beratung von Lesben, Schwulen, Bisexuellen und ihren Angehörigen (S. 104-120). Stuttgart: Klett-Cotta.

Wiesendanger, K. (2005). Vertieftes Coming-out. Göttingen: Vanderhoeck\&Ruprecht.

Wolf, G., \& Meyer, E. (2017). Sexuelle Orientierung und Geschlechtsidentität - (k)ein Thema in der Psychotherapie? Psychotherapeutenjournal, 2/2017, 130-139.

Wolf, G., Fünfgeld, M., Oehler, R., \& Andrae, S. (2015). Empfehlungen zur Psychotherapie und Beratung mit lesbischen, schwulen und bisexuellen Klient_innen. Verhaltenstherapie \& psychosoziale Praxis, 47(1),21-48.

Hinweis des Verlags Der Verlag bleibt in Hinblick auf geografische Zuordnungen und Gebietsbezeichnungen in veröffentlichten Karten und Institutsadressen neutral. 